\title{
Dynamic transcriptomes of resistant and susceptible peach lines after infestation by green peach aphids (Myzus persicae Sülzer) reveal defence responses controlled by the Rm3 locus
}

Liang Niu ${ }^{1,2+}$, Lei Pan ${ }^{2 \dagger}$, Wenfang Zeng ${ }^{2}$, Zhenhua Lu², Guochao Cui ${ }^{2}$, Meili Fan², Qiang Xu' ${ }^{1}$ Zhiqiang Wang ${ }^{2 *}$ and Guohuai $\mathrm{Li}^{1 *}$

\begin{abstract}
Background: The green peach aphid (GPA), Myzus persicae (Sülzer), is a widespread phloem-feeding insect that significantly influences the yield and visual quality of peach [Prunus persica (L.) Batsch]. Single dominant gene $(R m 3)$-based resistance provides effective management of this invasive pest, although little is known about the molecular responses of plants to GPA feeding.

Results: To illustrate the molecular mechanisms of monogenic resistance in peach to young tissue-infecting GPAs, aphid-resistant/aphid-susceptible peach lines from a segregating population with $R \mathrm{~m} 3 / \mathrm{rm} 3$ and $\mathrm{rm} 3 / \mathrm{rm} 3$ genotypes were infested with GPAs for 3 to $72 \mathrm{~h}$. Transcriptome analysis of the infested tissues identified 3854 differentially expressed genes (DEGs). Although the majority of the DEGs in the resistant line also responded to aphid attack in the susceptible line, the overall magnitude of change was greater in the resistant line than in the susceptible line. The enriched gene ontology of the 3854 DEGs involved in plant defence responses included redox situation, calcium-mediated signalling, transcription factor (e.g., WRKY, MYB, and ERF), MAPK signalling cascade, phytohormone signalling, pathogenesis-related protein, and secondary metabolite terms. Of the 53 genes annotated in a $460 \mathrm{~kb}$ interval of the $\mathrm{rm} 3$ locus, seven genes were differentially expressed between the aphid-resistant and aphid-susceptible peach lines following aphid infestation.

Conclusions: Together, these results suggest that the Rm3-dependent resistance relies mainly on the inducible expression of defence-related pathways and signalling elements within hours after the initiation of aphid feeding and that the production of specific secondary metabolites from phenylpropanoid/flavonoid pathways can have major effects on peach-aphid interactions.
\end{abstract}

Keywords: Rm3 locus, Peach, Myzus persicae (Sülzer), Innate immunity, Transcriptome analysis, Aphid resistance

\footnotetext{
* Correspondence: liguohuai@mail.hzau.edu.cn; wangzhiqiang@caas.cn

${ }^{\dagger}$ Liang Niu and Lei Pan contributed equally to this work.

'Key Laboratory of Horticultural Plant Biology, Ministry of Education,

Huazhong Agricultural University, Wuhan 430070, China

${ }^{2}$ Key Laboratory of Fruit Breeding Technology of Ministry of Agriculture,

Zhengzhou Fruit Research Institute, Chinese Academy of Agricultural

Sciences, Zhengzhou 450009, China
}

(c) The Author(s). 2018 Open Access This article is distributed under the terms of the Creative Commons Attribution 4.0 International License (http://creativecommons.org/licenses/by/4.0/), which permits unrestricted use, distribution, and reproduction in any medium, provided you give appropriate credit to the original author(s) and the source, provide a link to the Creative Commons license, and indicate if changes were made. The Creative Commons Public Domain Dedication waiver (http://creativecommons.org/publicdomain/zero/1.0/) applies to the data made available in this article, unless otherwise stated. 


\section{Background}

The green peach aphid (GPA) (Myzus persicae Sülzer), one of the most generalist aphid species, is capable of feeding on a wide range of food and ornamental crops from over 40 plant families in temperate regions and commonly uses peach, Prunus persica (L.) Batsch, as a primary host in spring [1]. The GPA ingests phloem sap from the host plant's sieve elements through narrow mouthparts called stylets [2]. Aphid feeding causes minimal damage to the hosts compared with the feeding of chewing insects. However, aphid infestation causes heavy damage to trees due to the penetration of the leaves by the stylet, which is responsible for leaf curling, heavy breakdowns in shoot growth, reduced fruit quality and fruit malformation [3]. GPA infestation causes further harm by spreading viruses, including the plum pox potyvirus (PPV), which is the world's most serious Prunus species disease [4]. Spraying insecticides is the primary method of controlling GPAs in peach orchards, although spraying results in increased production costs and environmental problems. Continuous use of insecticides has led to resistance to most classes of insecticides [5]. In addition, beneficial insects can be negatively affected by the continual usage of chemical insecticides [6].

To overcome the threat of GPAs, it is important to understand the peach immune system. Two layers of plant immunity are well defined: pattern-triggered immunity (PTI) and effector-triggered immunity (ETI) [7]. As the first line of the innate immune response, PTI is triggered by the perception of herbivore-associated molecular patterns (HAMPs) in the case of herbivory and microbe-associated molecular patterns (MAMPs) or pathogen-associated molecular patterns (PAMPs) in the case of infection by cognate plasma membrane-localized pattern recognition receptors (PRRs) [8]. Examples of HAMPs detected by plants include nematode pheromones (ascarosides), which are conserved among nematodes [9], and other components found in insect oral secretions (proteins, fatty acid-amino acid conjugates, sulphur-containing fatty acids) [10]. Plants recognize MAMPs/HAMPs and then initiate PTI with various defence responses, including the production of reactive oxygen species (ROS), deposition of callose, and reprogramming of the transcriptome to activate defences. [11]. To overcome this first line of defence pathways, adapted pathogens or herbivores can release may types of effectors that inhibit PTI into plant cells [7]. ETI, another pathogen-sensing mechanism mediated by plant resistance (R) proteins, can activate strong immune responses against pathogens and pests [12]. Generally, ETI is associated with strong immune responses, typically including the programmed cell death of plant cells at infection sites, which is called a hypersensitive response (HR) [13].
Host plant resistance seems to be the most favourable pest management method for environmental, economic, and social reasons [14]. In the past few years, five loci conferring resistance to phloem-feeding insects have been cloned. These loci include the tomato $\mathrm{Mi}-1.2$ gene [15] and the melon (Cucumis melo) Vat gene, which confer resistance to the aphids Macrosiphum euphorbiae and Aphis gossypii, respectively [16], and the rice genes Bph3, Bph14, and Bph26, which confer resistance to brown planthopper (Nilaparvata lugens) [17]. Five GPA-resistant genotypes from peach were identified in previous studies from the Institut National de la Recherche Agronomique, France (INRA); of these genotypes, two peach cultivars, "Weeping Flower Peach" and "Rubira", were controlled by a single dominant gene, i.e., $R m 1$ and $R m 2$ ( $\mathrm{Rm}$ for resistance to $M$. persicae), respectively [18-20]. Both genotypes showed a strong antixenosis-type resistance that prevented plant colonization by aphids; most GPAs left the plants within the first week following infestation, and approximately half left within the first 2 days [18]. In addition, reddish hypersensitive-like necrotic spots appeared at the puncture point on the apices of both genotypes within 23 days after the infestation [18]. A third antixenosis-type resistance genotype, which was also controlled by the single dominant gene $R m 3$, was identified in "Fen Shouxing" ( $P$. persica var. densa Makino) by our recent study [21]. Interestingly, although induced systemic resistance was only demonstrated for Rm2-type resistance $[19,20], R m 1, R m 2$ and $R m 3$ were mapped to the same narrow region (approximately $3 \mathrm{Mb}$ ) in chromosome 1 of the peach genome [22-24], and whether the GPA resistances conferred by "Weeping Flower Peach", "Rubira" and "Fen Shouxing" are controlled by one distinct locus is still a question.

Plant defences against phloem-feeding insects involve multiple signalling cascades and metabolic pathways, and molecular genetic studies on the model plant Arabidopsis thaliana have demonstrated that LRR family receptor-like kinases, calcium signalling proteins, hormone synthesis and signalling, reductant/oxidant (redox) signalling, transcription factors, and secondary metabolic pathways are at least partially activated by phloem feeding [25]. The influences of phloem-feeding insects, such as aphids, on plant transcriptomes have been well studied in susceptible and resistant plants in many species, including Arabidopsis, tomato, soybean, grape, rice, wheat, and maize [25]. However, there is no information about the molecular mechanism of peach resistance to GPAs.

Foyer et al. [25] reviewed previous transcriptome profiling studies on plants exposed to phloem-feeding insect attack and reported that there is a lack of understanding of the dynamic interactions between hosts and aphids, especially for fruit trees such as peach. The present 
study aimed to investigate the global responses of peach to GPA infestation and identify the genes associated with these resistance responses by comparing the differential gene expression of Rm3-type resistant (Rm3/rm3) and susceptible $(\mathrm{rm} 3 / \mathrm{rm} 3)$ peach lines with RNA sequencing-based gene expression profiling analysis. The results of this study provide insights into the interactions between peach and $M$. persicae and identify transcriptomic changes in peach that may be associated with resistance to Myzus persicae.

\section{Results}

\section{Dynamic analysis of resistance phenotypes against GPAs in R36 and S38}

Young apterous GPA adults were placed on the partially expanded trifoliate leaves of the susceptible individual S38 and the resistant individual R36 from an F1 progeny. The number of hypersensitive-like necrotic reactions on the young stems and the number of adult aphids remaining on the plants were measured 1 week after the aphid infestation. Hypersensitive-like necrotic reactions, an indicator of the presence or absence of Rm3-controlled GPA resistance, did not appear in the tender stems of either genotype during the first 2 days and then occurred in R36; however, these reactions were absent in susceptible plants throughout the experiment (Fig. 1a, c). Aphid settlement was similar in resistant individual R36 and susceptible individual S38 over the first 2 days after aphid infestation (DAI, Fig. 1b), as the $12 \mathrm{~h}$ picture in Fig. 1c shows. On 2 DAI, the aphids remaining on S38 showed no decrease or a very slow decrease, whereas the aphid number on R36 showed a steady and continuous decrease from 0 DAI to 2 DAI (Fig. 1b). Thus, we hypothesize that the signalling pathways and downstream genes that mediate defence events in the first 3 days of aphid feeding may be crucial to the peach resistance mechanisms. To unravel the molecular mechanisms involved in Rm3-type aphid resistance, eight sampling points covering the 3-day time interval were selected for transcriptome analysis.

\section{RNA sequencing and analysis of shoot-tip transcriptomes}

To survey the transcriptomic dynamics that occur in response to aphid feeding, the partially expanded trifoliate leaves of two peach lines, i.e., R36 and S38, were infested with GPAs for $0,3,6,9,12,24,48$, or $72 \mathrm{~h}$. mRNA-seq samples were taken at the expected time points. For transcriptome sequencing, RNA was extracted from two biological replicates per sample and converted into cDNA libraries for sequencing. The average number of clean reads for each sample was 47.6 million, of which $88.72 \%$ mapped to the $P$. persica v2.0.a1 genome reference sequence [26], which encodes 26,873 annotated genes and 47,089 transcripts (Additional file 1). Read
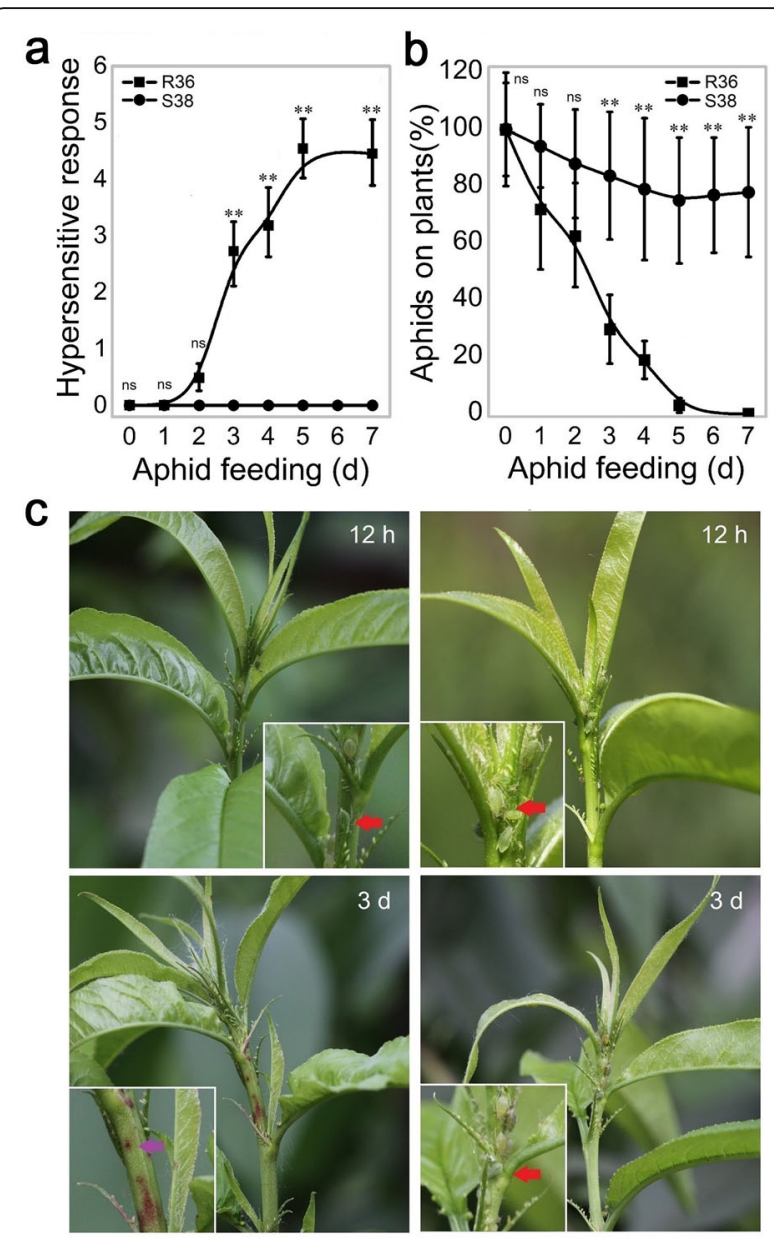

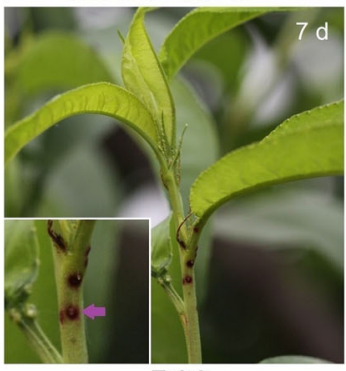

R36

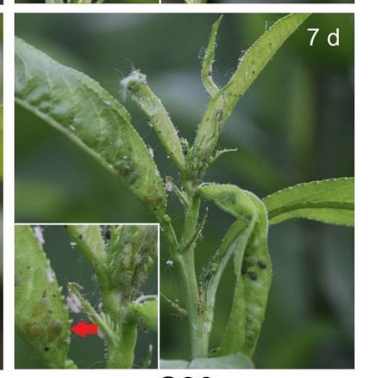

S38
Fig. 1 Peach hypersensitive-like reaction development and aphid settlement on R36 and S38. a Dynamic changes in the number of hypersensitive-like necrotic speckles after aphid feeding. $\mathbf{b}$ Percentage of adult aphids remaining on trees. Percentage calculated on 20 shoots, 15 aphids per shoot. c Representative pictures for peach hypersensitive-like reactions (purple arrows) and aphid (red arrows) settlement at $12 \mathrm{~h}, 3 \mathrm{~d}$, and $7 \mathrm{~d}$. For hypersensitive-like necrotic speckles and adult aphids remaining on trees, the results are the mean \pm SE of measurements for at least twenty shoots. Asterisks indicate statistically significant differences compared with 'S38' (A) or 'R36' (B) at similar days after aphid feeding using Student's t-test $\left({ }^{*} P<0.05,{ }^{*} P<0.01\right)$. ns indicates that there were no significant differences 
counts per gene were expressed as 'fragments per kilobase of transcript sequence per millions base pairs sequenced' (FPKM) mapped reads (Additional file 2). A total of 17,945 genes, approximately two-thirds of the annotated genes in the peach genome, were expressed in at least one sample (FPKM $\geq 2$ ), and more than half of the expressed genes $(13,088)$ were present in all samples.

\section{DEG analysis of R36 and S38 after aphid infestation}

To categorize the responses of differentially expressed genes (DEGs) to aphid infestation, the RNA-seq dataset was analysed using the DESeq R package (1.18.0) [27], which identified significant differences in gene expression by pairwise sample comparisons among the sixteen time points. Genes with significant differential expression $(P<$ 0.05 , false discovery rate [FDR]-adjusted) and a 2 -fold or greater change for at least one of the time points were selected, resulting in 3854 genes that were differentially expressed (Additional file 3). We also calculated the number of up- and down-regulated genes according to genotype. After the initiation of aphid feeding, hundreds of transcripts showed altered expression levels for each time point, up-regulated transcripts were much more abundant than down-regulated transcripts in the R36 plants, and the changes in gene expression peaked at 24 to $72 \mathrm{~h}$ after infestation (hai) (Fig. 2). Whereas DEG numbers peaked at 24 to 72 hai in R36, the greatest DEG number of induced genes was observed at 12 hai in S38 (Fig. 2). Generally, many more genes were differentially expressed in R36 tissues relative to $\mathrm{S} 38$, especially the up-regulated genes from 24 to 72 hai. The number of DEGs at later time points was markedly lower than that in S38 at 12 hai. Over time, the number of DEGs in S38 markedly decreased to 282 at 48 hai and 190 at 72 hai, indicating that the defence response of S38 started to slow down and that the leaves were already colonized at these time points by GPAs (Fig. 1). In contrast, the number of DEGs in R36 did not peak until 72 hai and increased significantly to 1438 at this time, which suggests that the duration of the defence response of R36 was longer and stronger than that of S38, and this peak in the number of DEGs in R36 revealed the significance of these periods. In addition, the analysis identified that more than half of the DEGs of S38 were differentially expressed in both cultivars (Fig. 2).

To assess transcriptome similarity among samples, principal component analysis (PCA) and hierarchical clustering were performed. Both analyses revealed two discrete groupings: one group consisted of the eight tissues from S38 and the first five time points of R36, and the other group consisted of the 24, 48 and 72 hai time points of R36 (Fig. 3), indicating that after 3 to $12 \mathrm{~h}$ of aphid feeding, the gene expression of the two genotypes was similar to that of the non-infested controls, and the greatest changes in gene expression occurred 1 day after the onset of aphid feeding in R36. The overall similarity of the PCA and hierarchical clustering results suggested that the aphid-induced gene expression changes induced changes in the transcriptome at the same or later time points in our experiments.

\section{Verification of the expression of some DEGs detected during aphid infestation}

Quantitative real-time PCR (qPCR) analysis was performed to validate our transcriptome profiling dataset by

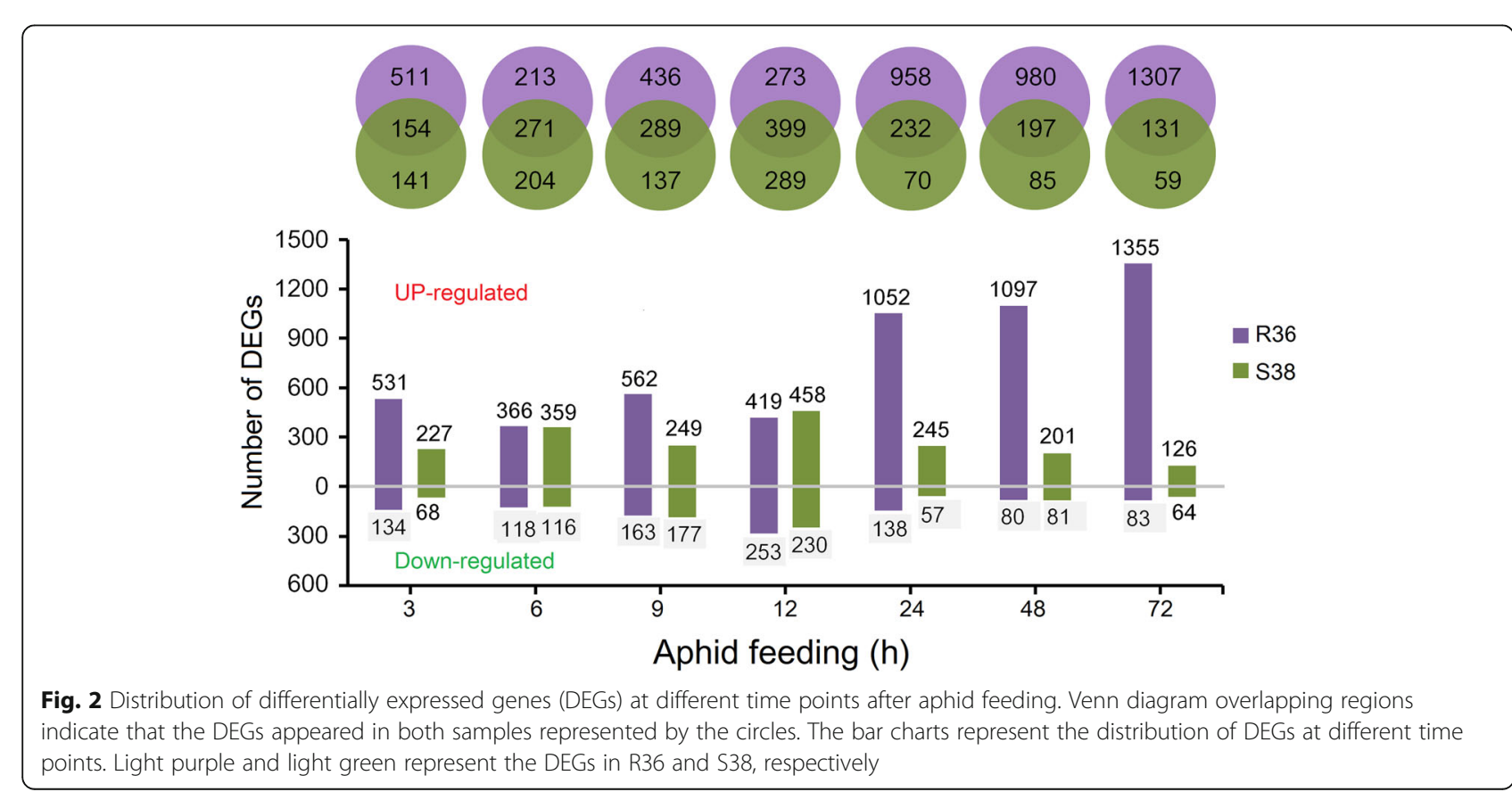




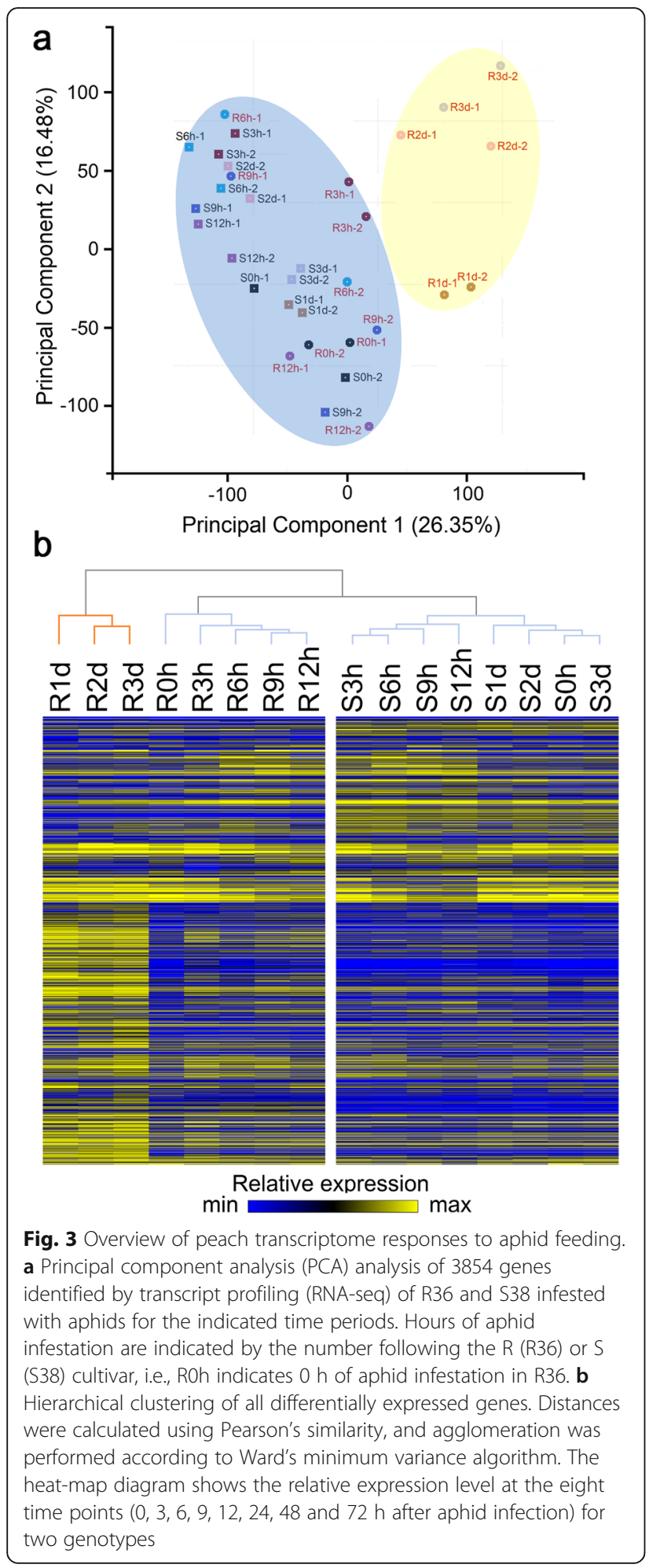

correlating the qPCR results with the standard RNA-seq data (presented in the Methods). We observed clear positive correlations between the qPCR and RNA-seq data for these two cultivars throughout the whole experimental period after GPA infection (Additional file 4).

\section{Cluster and gene ontology analysis of DEGs}

The significantly DEGs were input into TM4 software (http://www.tm4.org) for K-means cluster analysis using Pearson correlation distances. Each cluster was represented by the average gene expression level of the genes showing similar response patterns to aphid herbivory. The expression patterns of 3854 DEGs were divided into 12 clusters based on K-means cluster analysis and were classified into five groups manually (Fig. 4). In group I, which was the largest expression profile with 1714 DEGs, the genes were relatively stable both before and after aphid infestation in S38 but were continuously up-regulated in R36 post-infestation. Group I genes were divided into four clusters. In cluster 1 (714 genes), most genes were stable at the all the time points, except for 12 hai, in the S38 line but were continuously up-regulated in the R36 line. Cluster 2 (408 genes) genes were up-regulated from 3 to 12 hai in S36, continuously up-regulated in R36 from 24 to 72 hai, and down-regulated at 24 hai in S36. In cluster 3 (411 genes) and cluster 4 (181 genes), the gene expression profiles did not exhibit any significant differences in S38 but were consistently up-regulated after 24 hai in R36. For the three clusters in group II, the expression profiles of cluster 5 (213 genes), cluster 6 (451 genes), and cluster 7 (184 genes) were similar in both the R36 and S38 lines; gene expression profiles peaked in both lines at 3 hai, 6 hai, or 9 hai in these three clusters. Group III (694 DEGs) gene profiles were also similar in both the R36 and S38 lines. Samples at all time points had similar expression patterns, and gene expression profiles were down-regulated in both lines at 3 hai or 9 hai for cluster 8 or cluster 9. Genes in group IV (485 genes) were stable throughout aphid infestation in both the R36 and S38 lines, with a higher expression level in S38 for cluster 10 (300 genes) and in R36 for cluster 11 (185 genes). In group V (113 genes), the genes were down-regulated from 0 to 24 hai in the R36 line and were subsequently up-regulated from 24 to 72 hai. However, in the S38 line, these genes were stable throughout aphid infestation. Overall, these gene expression differences may account for the contrasting resistance phenotypes in the two individuals from a segregating progeny.

To elucidate the biological processes involved in each gene expression group, overrepresentation analysis was performed using the package 'GOseq R'. Enrichment of gene ontology (GO) terms was analysed separately for the DEGs, and significant GO terms identified among the DEGs included many terms associated with plant defence responses, stress responses and biotic stimulus responses (Table 1). This analysis provided evidence that calcium signalling and ROS pathways, as well as the production of protein phosphorylation and transcription factors, were up-regulated. Similarly, the observed gene 


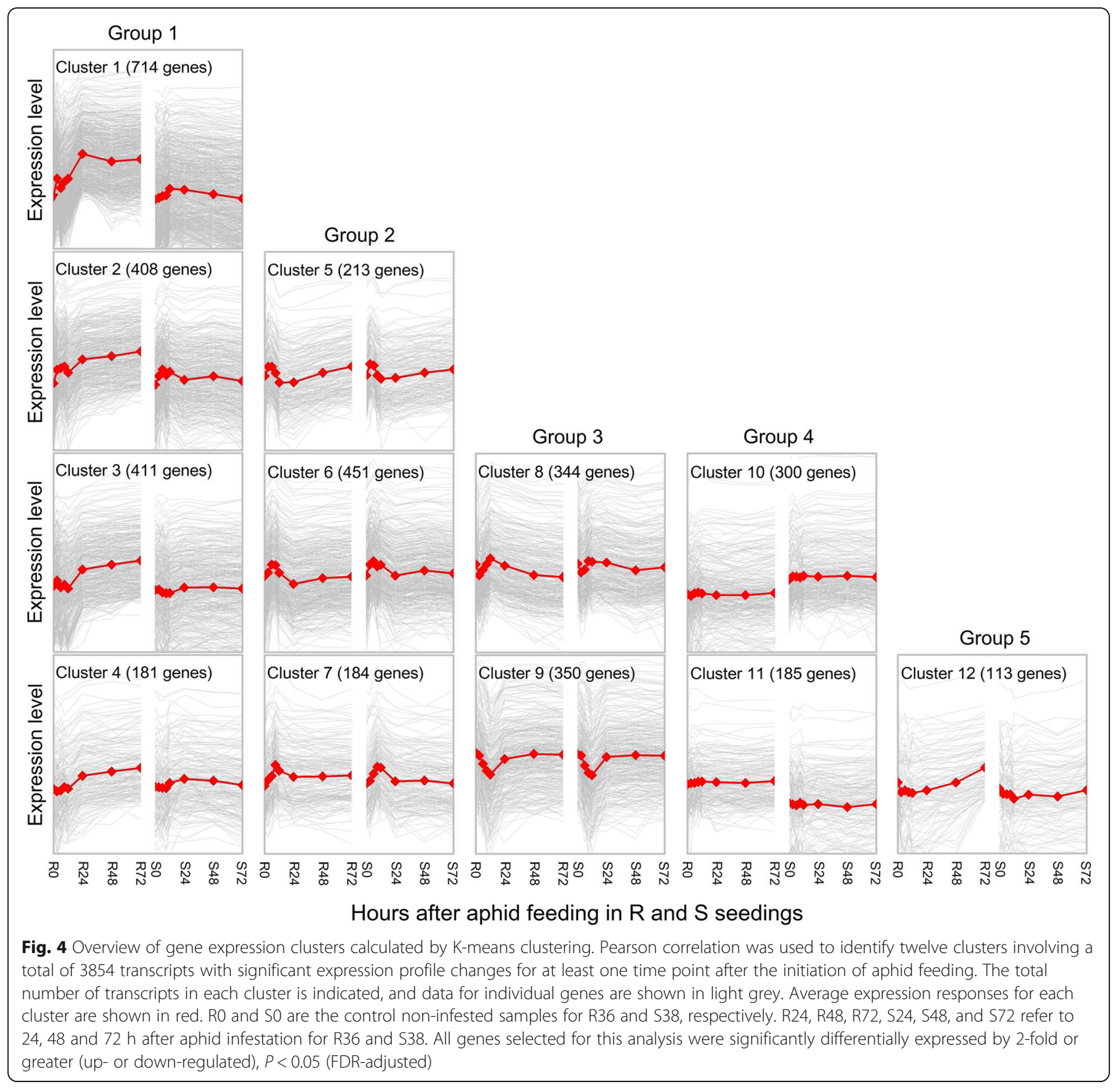

expression patterns indicate that the activation of metabolic processes occurs shortly after aphid feeding, and the most significantly enriched GO terms were metabolic process (GO:0008152), with 736 annotated genes, followed by single-organism process (GO:0044699). Other gene categories related to energy metabolism, including carbohydrate, polysaccharide, and glucan metabolic processes, were also overrepresented among the transcripts that were differentially expressed in response to aphid feeding. Conversely, genes involved in nucleobase-containing compound metabolic processes were overrepresented among the down-regulated genes and underrepresented among the up-regulated genes.
Together, these observations indicate that there is a shift in peach from primary metabolism to the production of defensive metabolites in response to GPA feeding.

\section{Overview of GPA-induced group I-type transcriptomic changes}

To understand the functional significance of the induced defence responses to GPA infestation, DEGs of group I were imported into the built-in biotic stress and secondary metabolism overview in MapMan software, which classifies the genes putatively involved in mediating biotic stress (pest or pathogen) responses [28]. Two pairwise comparisons between R36 and S38 were used in 
Table 1 Enriched gene ontology (GO) terms identified from the 3854 differentially expressed genes (DEGs) in the R36 and S38 during $24 \mathrm{~h}$ after aphid infestation

\begin{tabular}{|c|c|c|c|c|c|}
\hline GO accession & $\begin{array}{l}\text { Term } \\
\text { type }^{\mathrm{a}}\end{array}$ & Term description & P_Value & $\begin{array}{l}\text { DEG } \\
\text { item }\end{array}$ & $\begin{array}{l}\text { DEG } \\
\text { total }\end{array}$ \\
\hline \multicolumn{6}{|l|}{ Group 1} \\
\hline GO:0008152 & B & Metabolic process & 0.002 & 736 & 1257 \\
\hline GO:0044699 & B & Single-organism process & 0.002 & 534 & 1257 \\
\hline GO:0055114 & B & Oxidation-reduction process & 0.006 & 177 & 1257 \\
\hline GO:0006793 & B & Phosphorus metabolic process & 0.006 & 152 & 1257 \\
\hline GO:0006796 & B & Phosphate-containing compound metabolic process & 0.009 & 150 & 1257 \\
\hline GO:0006468 & B & Protein phosphorylation & 0.006 & 109 & 1257 \\
\hline GO:0006950 & B & Response to stress & 0.006 & 97 & 1257 \\
\hline GO:0006952 & B & Defence response & 0.001 & 41 & 1257 \\
\hline GO:0009607 & B & Response to biotic stimulus & 0.000 & 33 & 1257 \\
\hline GO:1901565 & B & Organonitrogen compound catabolic process & 0.006 & 19 & 1257 \\
\hline GO:0006505 & B & GPI anchor metabolic process & 0.014 & 17 & 1257 \\
\hline GO:0046149 & B & Pigment catabolic process & 0.011 & 11 & 1257 \\
\hline GO:0006787 & B & Porphyrin-containing compound catabolic process & 0.015 & 11 & 1257 \\
\hline GO:0033015 & B & Tetrapyrrole catabolic process & 0.015 & 11 & 1257 \\
\hline GO:0051187 & B & Cofactor catabolic process & 0.015 & 11 & 1257 \\
\hline GO:0031012 & c & Extracellular matrix & 0.030 & 19 & 1257 \\
\hline GO:0003824 & M & Catalytic activity & 0.010 & 688 & 1257 \\
\hline GO:0016491 & M & Oxidoreductase activity & 0.008 & 180 & 1257 \\
\hline GO:0004672 & M & Protein kinase activity & 0.009 & 113 & 1257 \\
\hline GO:0048037 & M & Cofactor binding & 0.023 & 75 & 1257 \\
\hline GO:0001071 & M & Nucleic acid binding transcription factor activity & 0.006 & 68 & 1257 \\
\hline GO:0003700 & M & Transcription factor activity, sequence-specific DNA binding & 0.006 & 68 & 1257 \\
\hline GO:0046906 & M & Tetrapyrrole binding & 0.011 & 53 & 1257 \\
\hline GO:0020037 & M & Heme binding & 0.013 & 52 & 1257 \\
\hline GO:0016758 & M & Transferase activity, transferring hexosyl groups & 0.044 & 48 & 1257 \\
\hline GO:0043565 & M & Sequence-specific DNA binding & 0.027 & 39 & 1257 \\
\hline GO:0005509 & M & Calcium ion binding & 0.023 & 36 & 1257 \\
\hline GO:0030246 & M & Carbohydrate binding & 0.001 & 32 & 1257 \\
\hline GO:0001871 & M & Pattern binding & 0.001 & 23 & 1257 \\
\hline GO:0030247 & M & Polysaccharide binding & 0.001 & 23 & 1257 \\
\hline GO:0009378 & M & Four-way junction helicase activity & 0.045 & 19 & 1257 \\
\hline \multicolumn{6}{|l|}{ Group II } \\
\hline GO:0044710 & B & Single-organism metabolic process & 0.001 & 149 & 531 \\
\hline GO:0055114 & B & Oxidation-reduction process & 0.002 & 86 & 531 \\
\hline GO:0005975 & B & Carbohydrate metabolic process & 0.000 & 52 & 531 \\
\hline GO:0044262 & B & Cellular carbohydrate metabolic process & 0.000 & 21 & 531 \\
\hline GO:0005976 & B & Polysaccharide metabolic process & 0.000 & 17 & 531 \\
\hline GO:0006073 & B & Cellular glucan metabolic process & 0.001 & 13 & 531 \\
\hline GO:0005618 & c & Cell wall & 0.005 & 13 & 531 \\
\hline GO:0030312 & c & External encapsulating structure & 0.008 & 15 & 531 \\
\hline GO:0003824 & M & Catalytic activity & 0.024 & 304 & 531 \\
\hline GO:0016491 & M & Oxidoreductase activity & 0.000 & 91 & 531 \\
\hline GO:0016757 & M & Transferase activity, transferring glycosyl groups & 0.031 & 32 & 531 \\
\hline GO:0016758 & M & Transferase activity, transferring hexosyl groups & 0.002 & 30 & 531 \\
\hline GO:0046906 & M & Tetrapyrrole binding & 0.002 & 30 & 531 \\
\hline
\end{tabular}


Table 1 Enriched gene ontology (GO) terms identified from the 3854 differentially expressed genes (DEGs) in the R36 and S38 during $24 \mathrm{~h}$ after aphid infestation (Continued)

\begin{tabular}{|c|c|c|c|c|c|}
\hline GO accession & $\begin{array}{l}\text { Term } \\
\text { type }^{\mathrm{a}}\end{array}$ & Term description & P_Value & $\begin{array}{l}\text { DEG } \\
\text { item }\end{array}$ & $\begin{array}{l}\text { DEG } \\
\text { total }\end{array}$ \\
\hline GO:0020037 & M & Heme binding & 0.007 & 28 & 531 \\
\hline \multicolumn{6}{|l|}{ Group III } \\
\hline GO:1901360 & B & Organic cyclic compound metabolic process & 0.003 & 133 & 463 \\
\hline GO:0046483 & B & Heterocycle metabolic process & 0.006 & 128 & 463 \\
\hline GO:0006725 & B & Cellular aromatic compound metabolic process & 0.006 & 128 & 463 \\
\hline GO:0006139 & B & Nucleobase-containing compound metabolic process & 0.000 & 127 & 463 \\
\hline GO:0009058 & B & Biosynthetic process & 0.049 & 126 & 463 \\
\hline GO:1901576 & B & Organic substance biosynthetic process & 0.034 & 123 & 463 \\
\hline GO:0044249 & B & Cellular biosynthetic process & 0.040 & 118 & 463 \\
\hline GO:0090304 & B & Nucleic acid metabolic process & 0.026 & 107 & 463 \\
\hline GO:1901362 & B & Organic cyclic compound biosynthetic process & 0.020 & 87 & 463 \\
\hline GO:0019438 & B & Aromatic compound biosynthetic process & 0.024 & 82 & 463 \\
\hline GO:0018130 & B & Heterocycle biosynthetic process & 0.026 & 82 & 463 \\
\hline GO:0034654 & B & Nucleobase-containing compound biosynthetic process & 0.001 & 81 & 463 \\
\hline GO:0006260 & B & DNA replication & 0.000 & 27 & 463 \\
\hline GO:0006753 & B & Nucleoside phosphate metabolic process & 0.038 & 18 & 463 \\
\hline GO:0009259 & B & Ribonucleotide metabolic process & 0.049 & 15 & 463 \\
\hline GO:0016747 & M & $\begin{array}{l}\text { Transferase activity, transferring acyl groups other than amino-acyl } \\
\text { groups }\end{array}$ & 0.000 & 25 & 463 \\
\hline GO:0016746 & M & Transferase activity, transferring acyl groups & 0.002 & 26 & 463 \\
\hline \multicolumn{6}{|l|}{ Group IV } \\
\hline GO:0043531 & M & ADP binding & 0.000 & 24 & 318 \\
\hline
\end{tabular}

the functional classification to understand the global changes in response to GPA feeding. The biotic stress response visualization presents substantial alteration in the transcriptomes of both the R36 and S38 genotypes after GPA infestation (Fig. 5a). A total of 681 transcripts belonging to signalling, hormone metabolism, secondary metabolites, cell wall modification, and changes in redox state, peroxidases, glutathione S-transferase (GST), beta glucanases, transcription factors, proteolysis and PRprotein related categories were found to be differentially expressed from 24 to 48 hai. More than 90 secondary metabolism pathway genes were identified in the group I genes, including phenylpropanoid, flavonoid, glucosinolate, lignan and terpenoid pathway genes (Fig. 5b). The annotation details and expression intensities of these differentially expressed transcripts mapped to the biotic stress pathway are presented in Additional file 5.

\section{Candidate gene analysis of the $r m 3$ locus in chromosome} 1

The Rm3 locus was placed within a 460-kb interval (Pp01:45.66 46.12 Mb) on chromosome 1 in our previous study [24]. The published peach genome was sequenced using the aphid-susceptible peach cv. Lovell [29], which does not have Rm3-type resistance.
Fifty-three genes were annotated in the 460-kb interval containing the rm3 locus in Lovell, and thirty-six of these genes were expressed (FPKM > 2) in the two genotypes at least one time point (Fig. 6). Based on gene annotation, four genes (Prupe.1G562000, Prupe.1G564100, Prupe.1G562100, and Prupe.1G564300) out of the thirty-six expressed genes may be involved in Rm3-type GPA resistance. However, the transcriptome analysis showed that only eleven of these genes had differential expression patterns in the leaves of R36 and S38 after GPA feeding (Additional file 6). The Prupe.1G564300 gene (WRKY DNA-binding protein 9) was the only identified candidate gene based on gene annotation and gene expression analysis.

\section{Discussion}

The genetics of peach resistance to aphids have been studied in detail. However, genomic research of peach-GPA interactions lags far behind. Although $M$. persicae is a pest of 40 different host plant families (including Brassicaceae, Solanaceae, Rosaceae, and Fabaceae), biotic stress responses following infestation by this generalist phloem-feeding insect have been established only for the model plants Arabidopsis and Solanum stoloniferum [25]. The main goal of this study was to 


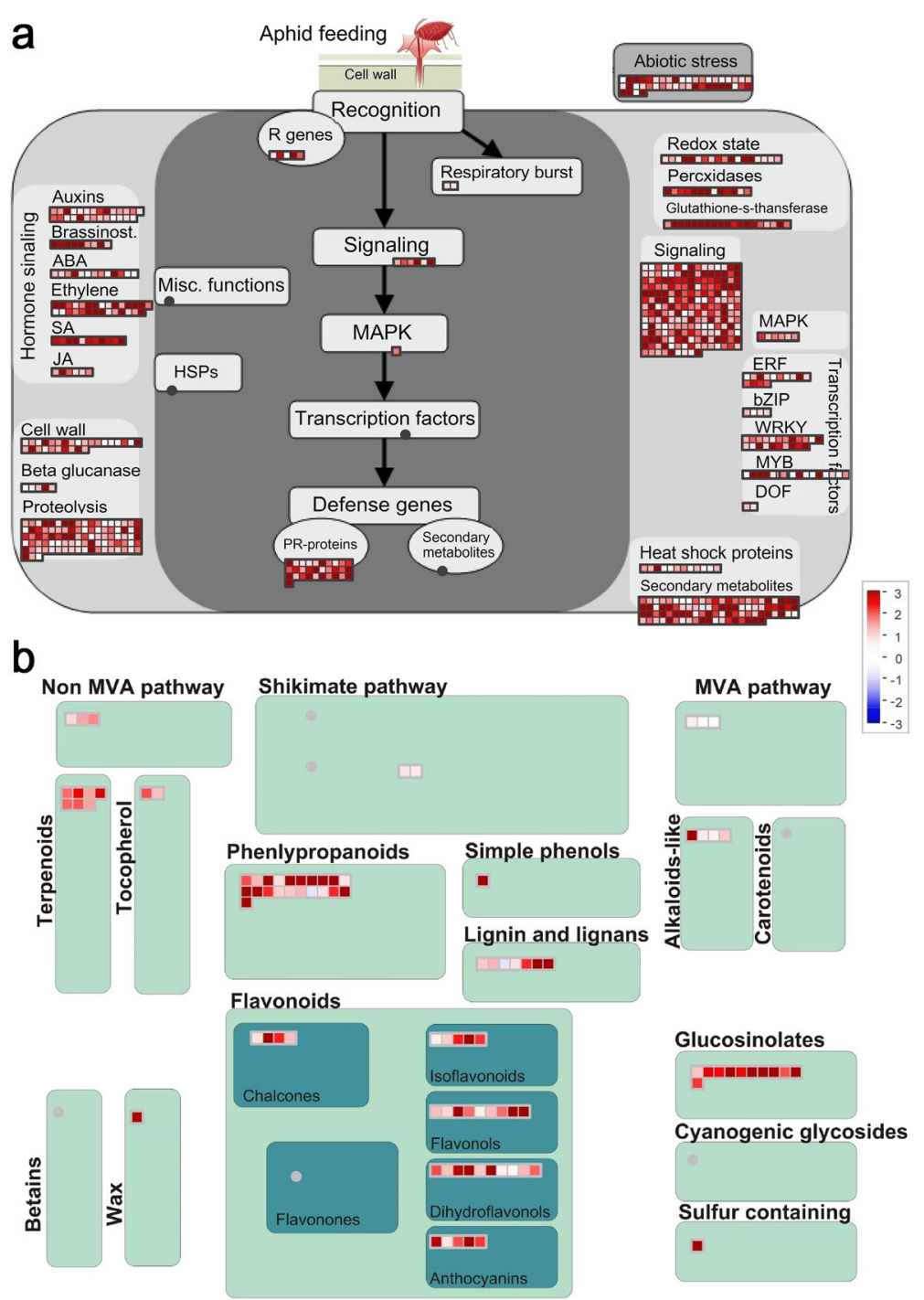

Fig. 5 Overview of group I transcripts assigned MapMan biotic stress (a) and secondary metabolism (b) terms at $24 \mathrm{~h}$ after GPA infestation in the resistant line (R36) compared with the susceptible line (S38). The log2-fold changes in the transcript levels were used for the analysis, and the colour scheme on the scale indicates the nature of gene expression; blue means "overexpression in S38", and red means "overexpression in R36". Each individual box represents a transcript classified under functional categories (BINs) defined by a MapMan biotic stress pathway

understand the molecular mechanism underlying the dominant resistance to $M$. persicae linked to $R m 3$ [21, 24]. In the present study, two lines from an F1 progeny, R36 and S38, segregating for $R m 3$ resistance traits in the bottom of chromosome 1 were compared using high-throughput sequencing technology. This approach allowed us to compare the changes in gene expression under $M$. persicae infection in the resistant and susceptible genotypes and to identify genes that were specifically induced in the resistant cultivar in response to infection.

\section{Aphid resistance is activated in R36}

The different transcriptomes of two peach genotypes were evaluated both before and after GPA infestation
(Fig. 1) using high-throughput Illumina sequencing technology. These peach lines differ in their resistance to $M$. persicae (Sülzer) controlled by the $R m 3$ locus at the bottom end of chromosome 1 [24] but are genetically related via the use of GPA-resistant lines from the parent wild peach material "Fen Shouxing". The aphid-resistant line R36 showed stronger antixenosis resistance than S38, which was fully susceptible to GPAs (Fig. 1). Hypersensitive-like necrotic reactions in the form of reddish or yellowish spots and rejected aphid settlement were observed in R36 but not in S38, while in S38, the apical stems and leaves were fully colonized by GPAs, and the considerable GPA invasion ultimately led to leaf curl. These results suggest that the $R m 3$ locus was 


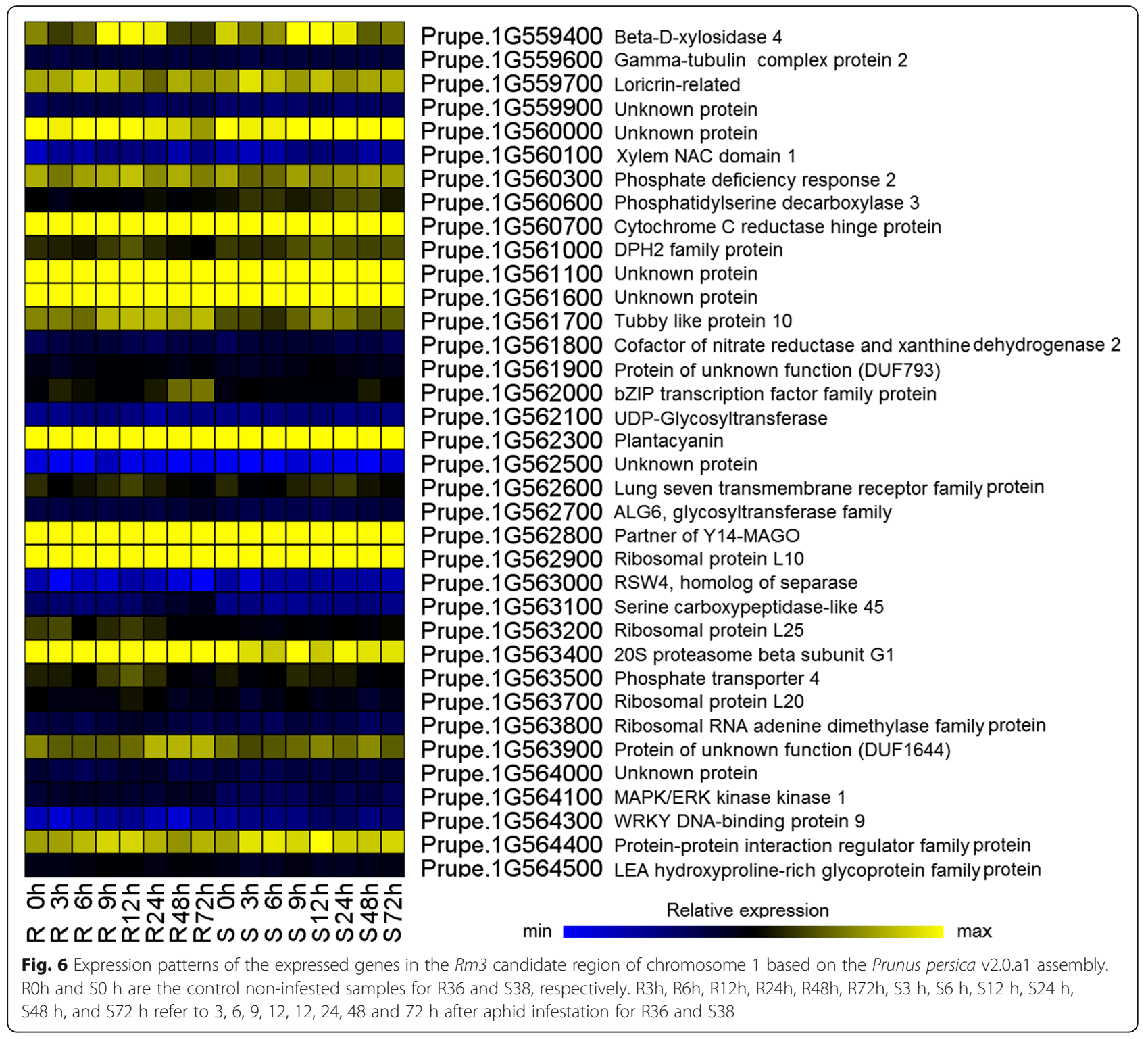

sufficient to confer a monogenic resistance to aphids that was associated with the HR reaction and GPA escape behaviour, which is similar to the dominant genetic resistance established for both "Weeping Flower Peach" [30] and "Rubira" [3].

Although this common defence-like response indicates that both lines can perceive M. persicae (Sülzer), the susceptibility of S38 clearly shows that the response of S38 is not sufficient to control this aphid. A comparison of the DEGs between R36 and S38 provided clues to the mechanisms that may explain the Rm3-type GPA resistance. To unravel the molecular mechanisms involved in this resistance, the transcriptomes of both lines were compared between non-infested plants and plants sampled from 3 to 72 hai, a key infection time interval in which the reddish or yellowish hypersensitive-like necrotic spots appeared on the apices at the puncture points and more than half of aphids left the resistant plants (Fig. 1). Overall, 3854 DEGs were identified. These DEGs are considered to encompass the most relevant genes for GPA defence because they include not only DEGs between the aphid-resistant and aphid-susceptible lines at eight key time points after aphid feeding but also DEGs between infested and non-infested plants at the same time point within R36 or S38, which account for aphid defence responses (in R36) that may not be captured by a direct comparison between R36 or S38. There were markedly more up-regulated DEGs than down-regulated DEGs in the comparisons between the aphid-exposed samples and the controls in both genotypes. Additionally, the number of up-regulated DEGs in the R36 genotype was higher 
than that in the S38 genotype (Fig. 4). Taken together, these results suggest that aphid infestation resulted in more activated than repressed genes in both the R36 and S38 genotypes, and the genes involved in defensive reactions to aphid infestation were activated at higher levels in the R38 genotype than in the S38 genotype.

\section{The $R m 3$ locus is associated with the induced expression of defence and signalling genes}

Among the genes that were induced more strongly in the resistant peach genotype than the susceptible peach genotype, we detected a significant enrichment in functional classes directly related to biotic stress resistance. These included genes associated with crucial biological processes, such as oxidation-reduction process, protein phosphorylation, stress response, defence response, biotic stimulus response and organonitrogen compound catabolic process terms (Table 1). The most significant up-regulation was observed for important protein families including WRKY transcription factors, ERF transcription factors, MYB transcription factors, PR proteins, and several secondary metabolism biosynthetic enzymes that play important roles in GPA resistance ((Fig. 5). Among these proteins, WRKYs are well known for regulating plant abiotic and biotic stress tolerance. These WRKY TFs specifically bind the cis-elements (TTGACC/T; W-box) in the promoter regions of their target genes, and the role of TFs in plant defences against pathogens and herbivores is well known. In rice, silencing OsWRKY45 reduced the feeding, oviposition preference, and survival rate of $N$. lugens [31]. Arabidopsis WRKY33 plays an important role in resistance to necrotrophic pathogens, and transgenic plants constitutively expressing the WRKY33 gene are more resistant than wild-type plants to necrotrophic pathogens [32]. We also observed the up-regulation of this gene family in peach in response to $M$. persicae infection, and the observed changes were stronger in the resistant line than in the susceptible line (Fig. 5). The induction of WRKY TF expression was more pronounced in resistant genotypes under $M$. persicae infection than in susceptible genotypes under $M$. persicae infection. Moreover, under stress conditions, the WRKY TF expression level was significantly higher in the resistant line than in the susceptible line, whereas under control conditions, there was no significant difference between the resistant and susceptible genotypes. In our case, the defence strategy conferred by $R m 2$ led to induced defences, which are efficient in terms of plant energetics and are economic and effective ways to protect plants from damage, allowing plants to quickly respond to aggressive and fast-spreading pathogens. Special attention should be paid to these genes in future searches for resistance gene candidates.

\section{Secondary metabolism pathways may be crucial for GPA resistance}

Plants have evolved sophisticated defence mechanisms to combat herbivore invasion; for example, plants produce specialized morphological structures and secondary metabolites and proteins that have toxic, repellent, and/ or antinutritional effects on herbivores [33]. Energy and nutrition are redistributed from growth and development to the synthesis of secondary metabolites that play a defensive role, resulting in protection from the attacker [34]. Cluster analyses showed that more than half of the DEGs (infested vs. non-infested) in both genotypes were differentially regulated between the two cultivars in response to M. persicae Sülzer infestation (Fig. 2). Genes in the phenylpropanoid, flavonoid, glucosinolate, lignan, alkaloid and terpenoid pathways were the most significantly induced and enriched at both time points, and more than 90 genes in secondary metabolism pathways were identified in the group I genes (Fig. 4). Phenylpropanoid metabolism transforms phenylalanine (Phe) into the majority of the phenolic compounds found in nature, including lignins, sinapate esters, stilbenoids and flavonoids, which play important roles in plants, especially as pigments and defence compounds [35]. Phenolic compounds such as flavonoids, lignans, stilbenes and tannins comprise a major class of inducible defence compounds in many woody species [33]. In peach, metabolome analyses have revealed that the accumulation of phenolic compounds (dicaffeoylquinic acids) in response to $M$. persicae infestation is correlated with Rm2-type GPA resistance [36]. In this study, GPA infestation resulted in the differential regulation of seven lignan pathway-related transcripts and thirty-three flavonoid pathway-related transcripts putatively associated with $M$. persicae infestation responses (Fig. 5b, Additional file 5). Alkaloids belong to a structurally diverse group of nitrogen-containing basic natural products with many targets and biological activities, such as interfering with nervous systems, the disruption of DNA synthesis and repair, and the inhibition of protein synthesis. The pea aphid (Acyrthosiphon pisum) is strongly deterred by indolizidine and quinolizidine alkaloids [37]. Interestingly, studies have shown that aphids may tolerate low concentrations of alkaloids, as the accumulation of these alkaloids provides a clear defensive benefit [38]. Glucosinolates are a group of anionic thioglucosides present in Brassicaceae [39] and do not seem to be toxic in the absence of their hydrolysing enzyme myrosinase. Upon plant tissue damage, glucosinolates co-occur with myrosinase and are rapidly hydrolysed to toxic isothiocyanates (mustard oils) [40]. After herbivore feeding, plants release high levels of mustard oil, which is a very effective defence against some generalist herbivores [41]. Aphids avoid activating this response by causing 
minimal damage to cells and thus consume and exude mostly intact glucosinolates with little negative effects [42]. Two aphid species, Brevicoryne brassicae and Lipaphis erysimi, sequester intact host plant glucosinolates and convert these glucosinolates to toxic mustard oils by using their own myrosinase [43, 44]; thus, glucosinolates have negligible or even beneficial effects on aphids. Terpenes are major components of plant volatile organic compounds (VOCs) and often play important roles in the plant defence by attracting enemies or predators of herbivores and repelling herbivorous insect feeding. The (E)- $\beta$-farnesene (EBF), an alarm pheromone towards aphids, is emitted by the $M$. persicae-infested host plant $[44,45]$. Based on the above analysis, although there are many DEGs in the metabolic pathways of terpenoids and glucosinolates, we believe that the secondary metabolites (including dicaffeoylquinic acids [36]) from the phenylpropanoid/flavonoid metabolic pathway are the most important defence responses for peach trees against GPAs.

\section{Gene expression analysis of the $r m 3$ locus}

In the past two decades, a number of loci conferring resistance to phloem-feeding insects have been identified and mapped. Including $R m 1, R m 2$ and $R m 3$, many mapped resistance loci show a strong homology or tight linkage with the NBS-LRR resistance protein gene family $[23,46-48]$, and five cloned $\mathrm{R}$ loci to date all control hemipteran insect resistance, suggesting that the $R m$-type gene is also a member of the NBS-LRR family [49]. NBS-LRR genes were up-regulated at high levels in host plants in response to biotic stresses [50-53]. The finely mapped genomic interval of $R m 3$ partly overlaps with the $(\sim 1.15 \mathrm{Mb})$ region of $R m 2$ (Pp01:45.08 $46.23 \mathrm{Mb})[22,24]$ and with the candidate genomic region $(2.88 \mathrm{Mb}$ ) of $R m 1$ (Pp01:43.62 $46.50 \mathrm{Mb}$ ) [23]. The mapped loci of the three type $R m$ genes are at the bottom of chromosome 1, which is flanked by NBS-LRR resistance gene analogues [54]. However, in this study, there was no differentially expressed $R$ gene from the finely mapped interval of Rm3-type genes (Fig. 6). As transcriptome data provide absolute rather than relative expression levels, the transcriptome can be used to identify the unexpressed genes in R36 and S38. Among the fifty-three genes annotated in the 460-kb interval containing the rm3 locus, thirty-six genes were expressed (Fig. 6, Additional file 6). These genes, especially the 7 genes with different expression levels in R36 and S38 after aphid infection, may be candidate genes for $R m 3$ (Fig. 6). Remarkably, only 1 out of the 7 genes was defence related; this gene is a WRKY DNA-binding protein gene (Prupe.1G564300). It is worth investigating the function of the two unknown genes because they may present a new explanation for the molecular mechanism of GPA resistance in peach. Considering the wild peach origin of the $R m 3$ gene, it is also plausible that this study may not be able to capture the regulatory gene in the rm3 locus because the genome of "lovell" does not possess antixenosis resistance to GPAs. We cloned a novel $\mathrm{R}$ gene from aphid-resistant peach lines derived from 'Shouxing Tao', and this gene co-segregated with Rm3-type aphid resistance (unpublished data). The protein encoded by this novel $\mathrm{R}$ gene could be responsible for the large number of DEGs, i.e., the disease resistance (R) genes, by controlling the specific ability to sense viral pathogen invasions and subsequently trigger a series of downstream immune responses [55]. Functional analysis of the novel $\mathrm{R}$ gene is currently underway to understand the mechanism of $R m 3$ resistance.

\section{Conclusions}

In the present study, a comparison between the expression profiles of the resistant peach line R36 and the susceptible peach line S38 during the early stage of $M$. persicae Sülzer- $P$. persica interaction revealed that the regulation of defence responses against $M$. persicae Sülzer was clearly different between the two genotypes. Genes mainly involving biotic stimulus and stress, the ROS scavenging system, TFs, PR proteins, and secondary metabolites including phenylpropanoids/flavonoids were more strongly up-regulated in the resistant genotype than in the susceptible genotype. Although it is not clear whether these genotype-specific differences in the transcript abundances of defence-related genes contribute to $M$. persicae Sülzer resistance in $P$. persica, these new discoveries support future experiments that may uncover the mechanisms responsible for $R m 3$-dependent GPA resistance in $P$. persica.

\section{Methods}

\section{Plant materials, aphid infestation, and sampling}

Two individuals from an F1 peach progeny were used in this study. The progeny was derived from a cross between 'Chun Mei', an aphid-susceptible peach (rm3/ rm3), and '01-29-23', an aphid-resistant peach (Rm3/ $r m 3)$, which had been segregated for $R m 3$ resistance traits. '01-29-23' was derived from 'Shouxing Tao' ( $P$. persica var. densa Makino), and genetic analysis indicated that the GPA resistance of 'Shouxing Tao' is controlled by a single dominant gene in linkage group 1 linked to the peach flesh colour (white or yellow) [21, 56]. This progeny consisted of 31 seedlings. Two lines, '07-15-36' (aphid-resistant, Rm3/rm3) and 07-15-38 (aphid-susceptible, $r m 3 / \mathrm{rm} 3$ ), referred to as R36 and S38, respectively, were used for aphid infestation and RNA-seq library construction. Trees were planted in a field at the ZFRI Experimental Station at Zhengzhou (Henan, China). The trees were planted in a $4 \times 2.0 \mathrm{~m}$ 
arrangement with drip irrigation and fertilizer applications as required. The test aphids were derived from a clonal culture of $M$. persicae originating from a single female collected on a peach tree grown in an orchard in the Zhengzhou Fruit Research Institute, China. This clone has been continuously reared on susceptible seedlings of peach plants (variety CN14) under parthenogenesis-inducing conditions of $20{ }^{\circ} \mathrm{C}$ and 16-h-light/8-h-dark photoperiod in a growth chamber [18]. For aphid infestation treatment, fifteen adult aphids were placed on the partially expanded trifoliate leaves of the susceptible and resistant individuals. Before sampling, the remaining aphids were carefully removed from the leaves. Five shoot tips were sampled at time 0 (no aphid infestation), 3, 6, 9, 12, 24, 48, and 72 hai. Collected materials were immediately frozen in liquid nitrogen and stored at $-80{ }^{\circ} \mathrm{C}$ until use.

\section{Aphid assays}

To assess the plant-colonizing ability of GPAs, fifteen apterous adults were deposited on the terminal shoots of the resistant and susceptible plants. The number of adults remaining alive on the plants over a period of 7 days was recorded. During this period, the number of hypersensitive-like necrotic reactions (a type of reddish or yellowish spots) was also recorded. The parameters were collected from 20 shoots for each plant.

\section{RNA-seq library construction and high-throughput sequencing}

Total RNA from the peach shoots was extracted using the RNAprep Pure Plant Kit with on-column DNase digestion (Tiangen Biotech Co., Ltd., Beijing, China) according to the manufacturer's instructions. RNA purity and concentration were measured using a NanoPhotometer spectrophotometer (IMPLEN, CA, USA), and the RNA integrity was confirmed using the RNA Nano 6000 Assay Kit from the Bioanalyzer 2100 system (Agilent Technologies, CA, USA). A total of 32 RNA-seq libraries (two biological replicates at eight time points for both the aphid-resistant and susceptible peach lines) were constructed as described previously [57] using the NEBNext Ultra Directional RNA Library Prep Kit for Illumina (New England Biolabs, Ipswich, MA) following the manufacturer's recommendations, and index codes were added so sequences could be attributed samples. The clustering of the index-coded samples was performed on a cBot Cluster Generation System using the TruSeq PE Cluster Kit v3-cBot-HS (Illumina) according to the manufacturer's instructions. After cluster generation, the library preparations were sequenced on an Illumina HiSeq 4000 platform, and 150 bp paired-end reads were generated. The whole library construction and Illumina sequencing step was performed at
Novogene Bioinformatics Technology Co., Ltd., Beijing, China (www.novogene.cn).

\section{RNA-seq data analysis}

Clean data (clean reads) were obtained by removing reads containing adapters, reads containing ploy- $\mathrm{N}$ and low-quality reads from raw data with in-house Perl scripts. At the same time, the Q20, Q30 and GC content values of the clean data were calculated. All downstream analyses were based on high-quality clean data. Reference genome and gene model annotation files were downloaded from GDR (URL: https://www.rosaceae.org/ species/prunus_persica/genome_v2.0.a1) [29]. The reference genome index was built using Bowtie v2.2.3 [58], and paired-end clean reads were aligned to the reference genome using TopHat v2.0.12 [59]. Relative transcript abundance was calculated based on fragments per kilobase of transcript sequence per millions base pairs (FPKM) for biological replicates. HTSeq v0.6.1 was used to count the read numbers mapped to each gene [60], and the differential expression of genes was determined with Cuffdiff (v.2.1.1), with bias correction applied to all samples.

\section{RNA extraction, complementary DNA synthesis and quantitative real-time PCR}

To validate the RNA-seq data, the expression of three biological replicates of fourteen genes (approximately $0.5 \%$ of all differentially regulated genes) was investigated by qPCR. RNA was extracted using the RNAprep Pure Plant Kit (TIANGEN, Beijing, China) according to the manufacturer's instructions. RNA quality, purity, and concentration were assessed using a Nano-Drop 2000 spectrophotometer. First-strand complementary DNA was synthesized using the FastQuant RT Kit (TIANGEN, Beijing, China) according to the manufacturer's instructions. All gene-specific primers were designed according to EST sequences using Primer Premier 5.0 (Additional file 7). Amplification reactions were run in a 96 well plate on a LightCycler 480 (Roche) with a LightCycler 480 SYBR Green I Master Kit (Roche Molecular Biochemicals), as previously described [59] under the following conditions: $95{ }^{\circ} \mathrm{C}$ for $30 \mathrm{~s}$, followed by 45 cycles of denaturation at $95{ }^{\circ} \mathrm{C}$ for $10 \mathrm{~s}$, annealing at $60{ }^{\circ} \mathrm{C}$ for $10 \mathrm{~s}$ and extension at $72{ }^{\circ} \mathrm{C}$ for $15 \mathrm{~s}$. Amplifications were normalized to actin, and each reaction was performed with three biological replicates for each sample. The relative expression levels were determined as described previously [61].

\section{GO enrichment analysis and MapMan analysis}

To evaluate functional activities differentially represented in aphid-susceptible or aphid-resistant lines, we mapped the DEGs to known biological ontologies based 
on the GO project (http://www.geneontology.org/). Enrichment analysis was performed on these sets using the $\mathrm{R}$ package 'GOseq', which accounts for biases because of the over-detection of long and highly expressed transcripts [62]. Gene sets with $\leq 10$ genes were excluded from the analysis. We used the REVIGO web page [63] to summarize and remove redundant $\mathrm{GO}$ terms from the results. Only GO terms with a FDR $<0.05$ were used. REVIGO plots were obtained for two GO categories: biological processes and molecular functions. The GO project describes a biological process as a recognized series of events or molecular functions with a defined beginning and end and describes molecular functions as activities that occur at the molecular level, such as catalytic or binding activities. The DEGs were clustered based on their expression patterns by the Genesis K-means method [28].

MapMan [28] was used for data visualization. False colour imaging was performed on the $\log _{2}$-transformed RNA-seq data. RNA-seq data were the average values of all replicates for each time point.

\section{Additional files}

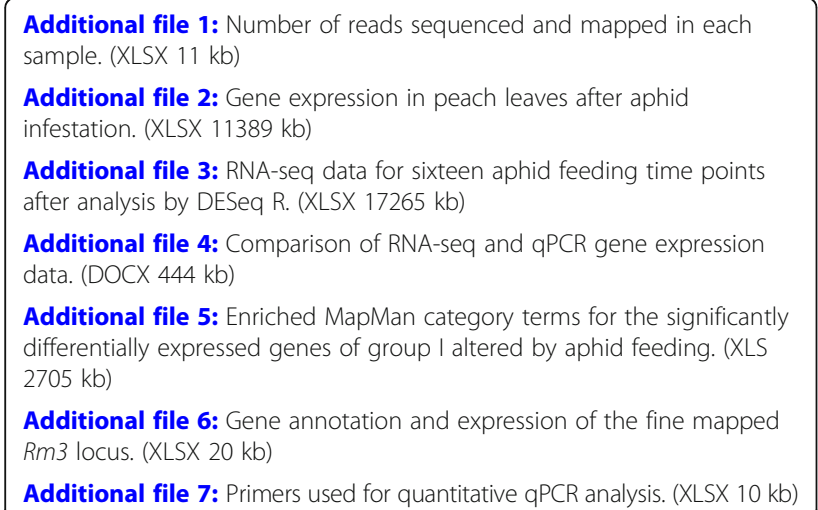

\section{Abbreviations}

DEGs: Differentially expressed genes; ETI: Effector-triggered immunity; GPA: Green peach aphid; HR: Hypersensitive response; MAMPs: Microbeassociated molecular patterns; PPV: Plum pox potyvirus; PR: Pathogenesisrelated; PRRs: Plasma membrane-localized pattern recognition receptors; PTI: Pattern-triggered immunity; ROS: Reactive oxygen species

\section{Acknowledgements}

We give thanks for the equipment and technical support provided by the scientific research shared service platform of ZFRI, CAAS.

\section{Funding}

This work is supported by the Agricultural Science and Technology Innovation Program (CAAS-ASTIP-2018-ZFRI), the National Natural Science Foundation of China (31701880), and the Central Public-interest Scientific Institution Basal Research Fund (1610192017703). The funding body had no role in the design of the study, the interpretation of data and the writing of the manuscript.

\section{Availability of data and materials}

The sequencing data are available in the NCBI Sequence Read Archive (project ID PRJNA454883).

\section{Authors' contributions}

LN, ZW and GL conceived the experiments; WZ and MF collected plant materials and conducted the experiments; $L N, L P, Q X$ and ZL performed the bioinformatic analysis; QX, GC and ZL analysed the data; and LN and LP wrote the manuscript text. All authors read and approved the final manuscript.

Ethics approval and consent to participate

Not applicable.

\section{Consent for publication}

Not applicable.

\section{Competing interests}

The authors declare that they have no competing interests.

\section{Publisher's Note}

Springer Nature remains neutral with regard to jurisdictional claims in published maps and institutional affiliations.

Received: 3 May 2018 Accepted: 31 October 2018

Published online: 28 November 2018

\section{References}

1. Blackman RL, Eastop VF. Aphids on the world's crops: an identification and information guide. Chichester: John Wiley \& Sons Ltd.; 2000.

2. Will T, Van Bel AJ. Physical and chemical interactions between aphids and plants. J Exp Bot. 2006;57(4):729-37.

3. Pascal T, Pfeiffer F, Kervella J, Lacroze JP, Sauge MH. Inheritance of green peach aphid resistance in the peach cultivar 'Rubira'. Plant Breed. 2002; 121(5):459-61.

4. Decroocq V, Foulongne M, Lambert P, Le Gall P, Mantin C, Pascal T, SchurdiLevraud V, Kervella J. Analogues of virus resistance genes map to QTLs for resistance to sharka disease in Prunus davidiana. Mol Gen Genomics. 2005; 272:680-9.

5. Foster SP, Devine G, Devonshire AL. Insecticide resistance: aphids as crop pests. Oxfordshire: CABl; 2007.

6. Christopher CG, Ramanaidu K, Astatkie T, Isman BM. Green peach aphid, Myzus persicae (Hemiptera: Aphididae), reproduction during exposure to sublethal concentrations of imidacloprid and azadirachtin. Pest Manag Sci. 2009;65(2):205-9.

7. Jones JDG, Dangl JL. The plant immune system. Nature. 2006:444(7117):323-9.

8. Zipfel C. Plant pattern-recognition receptors. Trends Immunol. 2014;35(7): 345-51.

9. Manosalva P, Manohar M, von Reuss SH, Chen S, Koch A, Kaplan F, Choe A, Micikas RJ, Wang X, Kogel KH, et al. Conserved nematode signalling molecules elicit plant defenses and pathogen resistance. Nat Commun. 2014. https://doi.org/10.1038/ncomms8795.

10. Wu J, Baldwin IT. Herbivory-induced signalling in plants: perception and action. Plant Cell Environ. 2009;32(9):1161-74.

11. Zipfel C, Robatzek S. Pathogen-associated molecular pattern-triggered immunity: veni, vidi...? Plant Physiol. 2010;154(2):551-4.

12. Jones JDG, Vance RE, Dangl JL. Intracellular innate immune surveillance devices in plants and animals. Science. 2016;354(6316):aaf6395.

13. Coll NS, Epple P, Dangl JL. Programmed cell death in the plant immune system. Cell Death Differ. 2011;18(8):1247.

14. Mundt CC. Durable resistance: a key to sustainable management of pathogens and pests. Infect Genet Evol. 2014;27:446-55.

15. Rossi M, Goggin FL, Milligan SB, Kaloshian I, Ullman DE, Williamson VM. The nematode resistance gene $\mathrm{Mi}$ of tomato confers resistance against the potato aphid. P Proc Natl Acad Sci USA. 1998;95(17):9750-4.

16. Dogimont C, Chovelon V, Pauquet J, Boualem A, Bendahmane A. The Vat locus encodes for a CC-NBS-LRR protein that confers resistance to Aphis gossypii infestation and A. gossypii-mediated virus resistance. Plant J. 2014; 80(6):993-04.

17. TamuraY HM, Yoshioka H, Yoshioka M, Takahashi A, Wu J, Sentoku N, Yasui H. Map-based cloning and characterization of a brown planthopper resistance gene BPH26 from Oryza sativa L. ssp. indica cultivar ADR52. Sci Rep-UK. 2014;4:5872. 
18. Sauge MH, Kervella J, Pascal T. Settling behaviour and reproductive potential of the green peach aphid Myzus persicae on peach varieties and a related wild Prunus. Entomol Exp Appl. 1998;89(3):233-42.

19. Sauge MH, Lacroze J, Poessel J, Pascal T, Kervella J. Induced resistance by Myzus persicae in the peach cultivar 'Rubira'. Entomol Exp Appl. 2002;102(1): 29-37.

20. Sauge M, Mus F, Lacroze J, Pascal T, Kervella J, Poessel J. Genotypic variation in induced resistance and induced susceptibility in the peach-Myzus persicae aphid system. Oikos. 2006;113:305-13.

21. Niu L, Lu ZH, Zeng WF, Cui GC, Pan L, Xu Q, Li GH, Wang ZQ. Inheritance analysis of resistance to green peach aphids (Myzus persicae Sülzer) for peach cultivar 'fen Shouxing' (Prunus persica var. densa). J Fruit Sci. 2016; 33(5):578-84.

22. Lambert P, Campoy JA, Pacheco I, Mauroux JB, Da Silva Linge C, Michelett D, Bassi D, Rossini L, Dirlewanger E, Pascal T, et al. Identifying SNP markers tightly associated with six major genes in peach [Prunus persica (L.) batsch] using a high-density SNP array with an objective of marker-assisted selection (MAS). Tree Genet Genomes. 2016;12(6):121.

23. Pascal T, Aberlenc R, Confolent C, Hoerter M, Lecerf E, Tuéro C, Lambert $\mathrm{P}$. Mapping of new resistance $(\mathrm{V} r 2, \mathrm{Rm} 1)$ and ornamental (Di2, pl) Mendelian trait loci in peach. Euphytica. 2017;213(6):132.

24. Zhang N, Lu Z, Cui G, Pan L, Zeng W, Niu L, Wang Z. Gene mapping of aphid-resistant for peach using SNP markers. Sci Agr Sinica. 2017;50(23): 4613-21.

25. Foyer $\mathrm{CH}$, Verrall SR, Hancock RD. Systematic analysis of phloem-feeding insect-induced transcriptional reprogramming in Arabidopsis highlights common features and reveals distinct responses to specialist and generalist insects. J Exp Bot. 2014;66(2):495-12.

26. Verde I, Jenkins J, Dondini L, Micali S, Pagliarani G, Vendramin E, Paris R, Aramini V, Gazza L, Rossini L, et al. The peach v2.0 release: high-resolution linkage mapping and deep resequencing improve chromosome-scale assembly and contiquity. BMC Genomics. 2017;18(1):225.

27. Anders $S$, Huber W. Differential expression analysis for sequence count data. Genome Biol. 2010;11(10):1-12.

28. Thimm O, Blasing O, Gibon Y, Nagel A, Meyer S, Kruger P, Selbig J, Muller LA, Rhee SY, Stitt M. MapMan: a user-driven tool to display genomics data sets onto diagrams of metabolic pathways and other biological processes. Plant J. 2010;37(6):914-39.

29. The International Peach Genome Initiative. The high-quality draft genome of peach (Prunus persica) identifies unique patterns of genetic diversity, domestication and genome evolution. Nat Genet. 2013;45(5):487-94.

30. Monet $\mathrm{R}$, Massonié $\mathrm{G}$. Déterminisme génétique de la résistance au puceron vert (Myzus persicae) chez le pêcher. Résultats complémentaires. Agron. 1994;13(3):177-82.

31. Huangfu J, Li J, Li R, Ye M, Peng K, Zhang T, Lou Y. The transcription factor oswrky45 negatively modulates the resistance of rice to the brown planthopper Nilaparvata lugens. Int J Mol Sc. 2016;17(6):697-711.

32. Zheng Z, Qamar SA, Chen Z, Mengiste T. Arabidopsis WRKY33 transcription factor is required for resistance to necrotrophic fungal pathogens. Plant J. 2006;48(4):592-605.

33. War AR, Paulraj MG, Ahmad T, Buhroo AA, Hussain B, Ignacimuthu S, Sharma HC. Mechanisms of plant defense against insect herbivores. Plant Signal Behav. 2012;7(10):1306-20.

34. Walling $\mathrm{LL}$, Kaloshian I. Plant-herbivore interactions in the era of big data: Management of Insect Pests to agriculture. Cham Heidelberg: Springer International Publishing; 2016.

35. Ververidis F, Trantas E, Douglas CJ, Vollmer G, Kretzschmar G, Panopoulos NJ. Biotechnology of flavonoids and other phenylpropanoid-derived natural products. Part I: chemical diversity, impacts on plant biology and human health. Biotechnol J. 2007:2(10):1214-34.

36. Poëssel JL, Sauge MH, Staudt M, Dufour C, Deborde C, Rahbé Y, Jackson B. PR-proteins and induced resistance against pathogens and insects: metabolite profiling and feeding bioassays suggest a major role for a Dicaffeoylquinic acid in induced resistance of peach to Myzus Persicae Aphid. In: OILB Meeting; 2011.

37. Dreyer DL, Jones KC, Molyneux RJ. Feeding deterrency of some pyrrolizidine, indolizidine, and quinolizidine alkaloids towards pea aphid (Acyrthosiphon pisum) and evidence for phloem transport of indolizidine alkaloid swainsonine. J Chem Ecol. 1985;11(8):1045-51.

38. Witte L, Ehmke A, Hartmann T. Interspecific flow of pyrrolizidine alkaloids: from plants via aphids to ladybirds. Naturwissenschaften. 1990;77:540-3.
39. Fahey JW, Zalcmann AT, Talalay P. The chemical diversity and distribution of glucosinolates and isothiocyanates among plants. Phytochemistry. 2001; 56(1):5-51.

40. Halkier BA, Gershenzon GJ. Biology and biochemistry of glucosinolates. Annu Rev Plant Biol. 2006;57(1):303-33.

41. Hopkins RJ, Van Dam NM, Van Loon JJ. Role of Glucosinolates in insectplant relationships and multitrophic interactions. Annu Rev Entomol. 2009; 54(1):57-83.

42. Kim J, Jander G. Myzus persicae (green peach aphid) feeding on Arabidopsis induces the formation of a deterrent indole glucosinolate. Plant J. 2010; 49(6):1008-19.

43. Francis F, Lognay $G$, Wathelet JP, Haubruge E. Effects of allelochemicals from first (Brassicaceae) and second (Myzus persicae and Brevicoryne brassicae) trophic levels on Adalia bipunctata. J Chem Ecol. 2001;27(2):243-56.

44. Dawson GW, Griffiths DC, Pickett JA, Wadhams L, Woodcock CM. Plantderived synergists of alarm pheromone from turnip aphid, Lipaphis (Hyadaphis) erysimi, (Homoptera, Aphididae). J Chem Ecol. 1987;13(7):1663-71.

45. Francis F, Lognay G, Haubruge E. Olfactory responses to aphid and host plant volatile releases: (E)-beta-farnesene an effective kairomone for the predator Adalia bipunctata. J Chem Ecol. 2004;30(4):741-55.

46. Francis F, Martin T, Lognay G, Haubruge E. Role of (E)---farnesene in systematic aphid prey location by Episyrphus balteatus larvae (Diptera: Syrphidae). Eur J Entomol. 2005;102(3):431-6.

47. Cevik V, King J. High-resolution genetic analysis of the $S d-1$ aphid resistance locus in Malus spp. Theor Appl Genet. 2002;105(2):346-54.

48. Gao L, Klingler JP, Anderson JP, Edwards OR, Singh KB. Characterization of pea aphid resistance in Medicago truncatula. Plant Physiol. 2008;146(3):996-09.

49. Lambert P, Pascal T. Mapping Rm2, gene conferring resistance to the green peach aphid (Myzus persicae, Sülzer) in the peach cultivar 'Rubira ${ }^{\otimes \prime}$. Tree Genetics \& Genomes. 2011;7(5):1057-1068.

50. Liu Y, Liu B, Zhu X, Yang J, Bordeos A, Wang G, Leach JE, Leung H. Finemapping and molecular marker development for Pi56(t), a NBS-LRR gene conferring broad-spectrum resistance to Magnaporthe oryzae in rice. Theor Appl Genet. 2013;126(4):985-98.

51. Zhang $C$, Chen $H$, Cai T, Deng $Y$, Zhuang R, Zhang N, Zeng $Y$, Zheng $Y$, Tang R, Pan R, et al. Overexpression of a novel peanut NBS-LRR gene AhRRS5 enhances disease resistance to Ralstonia solanacearum in tobacco. Plant Biotechnol J. 2017;15(1):39-55.

52. Zhu X, Lu C, Du L, Ye X, Liu X, Coules A, Zhang Z. The wheat NB-LRR gene TaRCR1 is required for host defence response to the necrotrophic fungal pathogen Rhizoctonia cerealis. Plant Biotechnol J. 2017;15(6):674-87.

53. He H, Zhu S, Zhao R, Jiang Z, Ji Y, Ji J, Qiu D, Li H, Bie T. Pm21, encoding a typical CC-NBS-LRR protein, confers broad-spectrum resistance to wheat powdery mildew disease. Mol Plant. 2018;11(6):879-82.

54. Ghelder CV. Esmenjaud D. TNL genes in peach: insights into the post-LRR domain. BMC Genomics. 2016;17(1):317.

55. Gururani MA, Venkatesh J, Upadhyaya CP, Nookaraju A, Pandey SK, Park SW. Plant disease resistance genes: current status and future directions. Physiol Mol Plant Pathol. 2012;78:51): 51-65.

56. Falchi R, Vendramin E, Zanon L, Scalabrin S, Cipriani G, Verde I, Vizzotto G, Morgante $M$. Three distinct mutational mechanisms acting on a single gene underpin the origin of yellow flesh in peach. Plant J. 2013;76(2):175-87.

57. Pan L, Zeng WF, Niu L, Lu ZH, Liu H, Cui GC, Zhu YJ, Chu JF, Li WP, Fang WC, et al. PpYUC11, a strong candidate gene for the stony hard phenotype in peach (Prunus persica L. Batsch), participates in IAA biosynthesis during fruit ripening. J Exp Bot. 2015;66(22):7031-44.

58. Langmead B, Salzberg SL. Fast gapped-read alignment with bowtie 2. Nat Methods. 2012;9(4):357-9.

59. Trapnell C, Pachter L, Salzberg SL. TopHat: discovering splice junctions with RNA-Seq. Bioinformatics. 2009;25(9):1105-11.

60. Anders S, Paul TP, Wolfgang H. HTSeq-a Python framework to work with high-throughput sequencing data. Bioinformatics. 2015;31(2):166-9.

61. Livak KJ, Schmittgen TD. Analysis of relative gene expression data using realtime quantitative PCR and the $2^{-\Delta \Delta C T}$ method. Methods. 2001;25(4):402-8.

62. Young MD, Wakefield MJ, Smyth GK, Oshlack A. Gene ontology analysis for RNA-seq: accounting for selection bias. Genome Biol. 2010;11(2):1-12.

63. Supek F, Bosnjak M, Skunca N, Smuc T. REVIGO summarizes and visualizes long lists of gene ontology terms. PLoS One. 2011;6(7):e21800. 